\title{
Agent based Bandwidth Reservation Routing Technique in Mobile Ad Hoc Networks
}

\author{
Vishnu Kumar Sharma \\ Department of CSE, JUET, India
}

\begin{abstract}
In mobile ad hoc networks (MANETs), inefficient resource allocation causes heavy losses to the service providers and results in inadequate user proficiency. For improving and automating the quality of service of MANETs, efficient resource allocation techniques are required. In this paper, we propose an agent based bandwidth reservation technique for MANET. The mobile agent from the source starts forwarding the data packets through the path which has minimum cost, congestion and bandwidth. The status of every node is collected which includes the bottleneck bandwidth field and the intermediate node computes the available bandwidth on the link. At the destination, after updating the new bottleneck bandwidth field, the data packet is feedback to the source. In resource reservation technique, if the available bandwidth is greater than bottleneck bandwidth, then bandwidth reservation for the flow is done. Using rate monitoring and adjustment methodologies, rate control is performed for the congested flows. By simulation results, we show that the resource allocation technique reduces the losses and improves the network performance.
\end{abstract}

Keywords- Mobile Ad hoc Networks (MANETs); Mobile Agents (MA); Total Congestion Metric (TCM); Enhanced Distributed Channel Access (EDCA); Transmission opportunity limit (TXOP).

\section{INTRODUCTION}

Mobile Ad Hoc networks:

The mobile ad hoc network is capable of forming a temporary network, without the need of a central administration or standard support devices available in a conventional network, thus forming a infrastructure-less network. In order to guarantee for the future, the mobile ad hoc networks establishes the networks everywhere. To avoid being an ideal candidate during rescue and emergency operations, these networks do not depend on the irrelevant hardware. These networks build, operate and maintain with the help of constituent wireless nodes. Since these nodes have only a limited transmission range, it depends on its neighboring nodes to forward packets [1].

\section{RESOURCE ALLOCATION AND ITS ISSUES IN MANET}

For the sake of improving and automating the quality of service of the networks, efficient resource allocation techniques are required. Resource allocation is carried out in a static manner on the hours to months scale of time in telecommunication networks. If traffic varies significantly, then resource allocated in the statical manner is inadequate or under-exploited. [2]

Wireless networks are emerging hastly and endlessly with the condition related to the rising transmission speeds, number

\author{
Dr. Sarita Singh Bhadauria \\ Department of Elex, MITS, India
}

of users and services. Owing to the huge number of the customers, the resource on the network has high requirement and competition. In addition, the necessity of the resources in a wireless networks varies depending on the network load and radio channel conditions. The resource reservation inefficiency causes high losses to the service providers and results in inadequate user proficiency. Hence active resource management system which has capability to make best use of resource is required. [3]

For the ad hov network application, the bandwidth reservation process are required for the real time flows. If the admission control is matched with the network characteristics, then reservations can avoid congestion occurence. Best effort traffic is not restricted to any scheme, and thus can intersect on the bandwidth share of the advantaged traffic resulting in assurance more delicate. The feasible solution to this difficulty will be assigning a constant bandwidth for best effort traffic. The solution doesn't take resources needed for the traffic or topology of the network into consideration. These solution frequently results in optimal use of the network resources. The another option is bandwidth allocation to best effort traffic depending on the topology and bandwidth existing in every mobile. [4]

The active nature of the MANET causes unpredicted intusion of attacks or faults which further results in seperation of the network, performance degradation, violation of the QoS requirements and more specifically disturb the bandwidth reservation. [10]

\section{PREVIOUS WORKS}

In paper [11], we proposed an agent based congestion control technique in MANET. In our technique, the node is classified in one of the four categories depending on whether the traffic belongs to background, best effort, video or voice AC respectively. Then MA estimates the total congestion metric by calculating the queue length and the channel contention and it is applied to the routing protocol to select the minimum congested route.

In paper [12] we proposed an agent based power control technique in MANET. In power control technique, the nodes are chosen based on the power level. The nodes with maximum power level are selected as listening nodes (LN) which will always be in active node and remaining nodes are selected as non-listening nodes (NLN) which will awake in periodic manner. The status of LN nodes keeps changing in every time cycle. The source transmits the data packets to the destination through the selected path. If the node receiving the packet is awake, the packet is transmitted to that node otherwise node 
checks for the nearest listening for transmitting the packet. In this manner, the packets are transmitted in hop-by-hop manner with reduced power consumption.

In our existing approaches, though mobile agents reduce the congestion and power, inefficient allocation of the resources may incur heavy losses to the service providers as well as poor user experience. Hence in our extension work, we are planning to include the agent based resource allocation in MANET.

\section{RELATED WORKS}

R.Gunasekaran et al [5] proposed the high-privileged and low-privileged architecture (HPLP) for Ad Hoc network for achieving optimal differentiated services for different classes of users. The new protocol, D-MACAW, was implemented. Among the various factors influencing the differentiated services, bandwidth reservation is only considered and different factors that can influence the efficiency of the bandwidth reservation are identified. The drawback of this proposed approach is that the complexity issues such as processing time, transaction time (latency), buffer management and memory utilization is not considered.

Kumar Manoj et al [6] proposed an algorithm that contains bandwidth calculation and slot reservation for mobile networks which could be applied to multimedia ad hoc wireless networks. Specially, the bandwidth information can be used to assist in performing the handoff of a mobile host between two base stations. Traffic flows with different QoS types have been considered. In addition to, standby routing enhances the performance in the mobile environment.

Rafael Guimarães et al [7] proposed a QoS reservation mechanism for multirate ad hoc wireless networks that allows bandwidth allocation on a per flow basis. By multirate they refer to those networks where wireless nodes are able to dynamically switch among several link rates. This allows nodes to select the highest possible transmission rate for exchanging data, independently for each neighbor. This reservation approach provides a feasible way to avoid congestion, guaranteeing, thus QoS requirements to ongoing connections.

Maria Canales et al [8] proposed an adaptive cross-layer architecture based on the cooperation between a QoS routing protocol and the MAC level. This joint operation allows to perform a distributed admission control capable of providing the required end-to-end QoS adapting the operation to the characteristic variant environment of MANETs. The proposed scheme has been designed a flexible parameters configuration that allows to adapt the system response to the observed grade of the mobility in the environment.

Wang Xiangli et al [9] proposed a distributed bandwidth reservation protocol (DBRP) for QoS routing in ad hoc networks. The protocol adopts a TDMA-based model, derives from AODV, refers to the idea of three slot states, and adopts two-time reservation and controlling-flooding scheme. The protocol takes both the hidden-terminal and exposed-terminal problems into account. And it can solve the simultaneous reservation of several paths. In addition, controlling-flooding method can effectively control routing overhead, and two-time reservation can improve request success rate.

\section{PROPOSED WORK}

\section{A. Overview}

The mobile agent from source starts forwarding the data packets through the path containing minimum cost, congestion and bandwidth availability. The packets upon reaching every intermediate node updates its list with the node information such as it's id, flag, power level, node activating counter, information about the neighbor node, cumulative assigned rates for incoming and outgoing flow and requested data rate stored in the bottleneck bandwidth $\left(\mathrm{BW}_{\mathrm{BN}}\right)$ field and the intermediate node computes the available bandwidth $\left(\mathrm{B}_{\mathrm{av}}\right)$ on the link. If $\mathrm{B}_{\mathrm{av}}>\mathrm{BW}_{\mathrm{BN}}$, then the node forwards the packet to the next node on the path. Else the node replaces $\mathrm{BW}_{\mathrm{BN}}$ with $\mathrm{B}_{\mathrm{av}}$ and proceeds to forward to the next node. When the data packet reaches the destination, $\mathrm{BW}_{\mathrm{BN}}$ field is copied to new packets and fedback to the source. The intermediate node updates its routing table with new $\mathrm{BW}_{\mathrm{BN}}$ value when the data packet is traversing towards the source. The source after receiving the data packet updates its routing table with the new $\mathrm{BW}_{\mathrm{BN}}$ value. If the $\mathrm{B}_{\mathrm{av}}>\mathrm{BW}_{\mathrm{BN}}$, then reservation of bandwidth for the flow can be proceeded. Otherwise, the $B W_{B N}$ is overwritten with the $\mathrm{B}_{\mathrm{av}}$. The rate control technique concentrates on rate monitoring and adjustment methodologies where the cumulative assigned rate for incoming and outgoing flow helps in rate adjustment.

\section{B. Available Bandwidth estimation}

Every node is in charge for estimating the available bandwidth on its link. For a given node,

Let $\mathrm{B}_{\mathrm{av}}=$ available bandwidth.

$\mathrm{L}=$ link capacity associated with one-hop neighbor $\mathrm{i}$.

ACA be the cumulative assigned rates for all incoming and outgoing flows.

Hence the sum of the assigned incoming and outgoing flow rates and available bandwidth on the link should be equal the capacity of the link i. This can be expressed as

$$
\mathrm{ACA}^{i j}+\mathrm{B}_{\mathrm{av}}=\mathrm{L}_{\mathrm{i}}
$$

The mobile agent from the source node forwards the data packet along a given path towards the destination. The data packet constitutes the requested bandwidth value stored in the bottleneck bandwidth filed. Each intermediate node is responsible for determining whether or not sufficient bandwidth is available on the local outgoing link to support the new flow request.

The link capacity is measured and available bandwidth is defined by

$$
\mathrm{B}_{\mathrm{avj}} \triangleq \max \left\{0, \mathrm{~L}_{\mathrm{j}}-\mathrm{ACA}^{i j}\right)
$$

\section{Resource allocation technique}

1) Entries of node's routing table 
Each node constitutes the routing table that includes the entries of its id, flag, power level, node activating counter, cumulative assigned rates for incoming and outgoing flow and requested data rate stored in the bottleneck bandwidth $\left(\mathrm{BW}_{\mathrm{BN}}\right)$ field.The amount of quantity of the routing table entries is found

based on the number on the active incoming and outgoing flows which is expressed as $n(n-1)$, where $n$ is the number of neighbors of the node.

The routing table also includes the following values

Assigned ACA ${ }^{i j}$ corresponding to incoming and outgoing flow.

Counter $\mathrm{CNT}_{\mathrm{ij}}$ for the number of bits that have arrived in the current measurement window.

Measured rate $\mathrm{CA}{ }^{i j}$ from the previous measurement window.

Every node is responsible for policing the incoming and outgoing flow to the cumulative assigned rate $\mathrm{ACA}^{i j}$. This measured rate $\mathrm{ACA}^{i j}$ helps in performing rate-adjustment.

The source node selects path with minimum power consumption and congestion as per previous paper (12)]. The following section describes the steps involved in the bandwidth reservation technique.

\section{2) 2. Steps Involved in Bandwidth Reservation} Step 1

The mobile agent from the source node forwards the data packet that contains the IP address of source and destination, flow ID and requested data rate stored in the $\mathrm{BW}_{\mathrm{BN}}$ field to the destination.

\section{$\underline{\text { Step } 2}$}

The intermediate node upon receiving the data packets determines the $\mathrm{B}_{\mathrm{av}}$ on its outgoing link.

\section{$\underline{\text { Step } 3}$}

If $\mathrm{B}_{\mathrm{av}}$ is greater than the $\mathrm{BW}_{\mathrm{BN}}$ value, then

Node forwards the packet to the next node on the path

Else

Node replaces the $\mathrm{BW}_{\mathrm{BN}}$ field with the value of $\mathrm{B}_{\mathrm{av}}$ and forwards the packet to next node.

End if

This process continues till the data packet reaches the destination.

Step 4

When the destination node receives the data packet, it copies the value of the $\mathrm{BW}_{\mathrm{BN}}$ to the new data packet and sent back to the source node using the reverse path.
Step 5

The intermediate node upon receiving the data packet updates its routing table with the new $\mathrm{BW}_{\mathrm{BN}}$ and then forwarded the packet to the next node.

\section{$\underline{\text { Step } 6}$}

The routing table is updated in the following way. Let ni (represented as in-hop) be the next node to which the new data packet will be sent and nj (represent as out-hop) be the node from which the packet was received.

If the routing table entry for incoming and outgoing flow already exists (i.e the flow is active).

Then current BWBN value in the new data packet is added to the reserved rate $\mathrm{CAij}$, associated with the incoming and outgoing flow.

Else

The routing table entry is created with an assigned rate value CAij (set equal to the BWBN value of the fedback data packet).

End if

This process continues till the data packet reaches the source.

Step 7

When the data packet reaches the source node, the source establishes the real-time flow based on the value of the $\mathrm{BW}_{\mathrm{BN}}$ field.

If the value of $\mathrm{B}_{\mathrm{av}}$ in source node is greater than or equal to the $\mathrm{BW}_{\mathrm{BN}}$ value in the packet

Then reservation of bandwidth for the flow can proceed

Else

The BWBN value in the new data packet is overwritten with the (smaller) value Bav.

end if

\section{Rate Monitoring and Adjustment}

In the rate monitoring strategy for a real time flow, the rate of flow is measured and compared with the assigned rate which is updated in the routing table. If the rate measured is lesser than the reserved rate by the sufficient margins, then the reserved rate is reduced by certain factor.

The traffic rate of a given flow during time interval $t$ can be measured by rate monitoring methodology. This is achieved by maintaining a counter that keeps the count value of the total number of bits arriving on an incoming and outgoing flow over a time $t$. As each packet arrives on a given flow (i,j), a counter $\mathrm{CNT}_{\mathrm{ij}}$ is incremented in terms of the size of the packets (in

bits). After lapse of time period $\mathrm{t}$, the measured rate $\mathrm{CA}^{i j}$ becomes

$$
\mathrm{CA}^{i j}=\mathrm{CNT}_{\mathrm{ij}} / \mathrm{t}
$$


The following step describes the rate adjustment strategy.

$$
\begin{aligned}
& \text { If }\left(\mathrm{ACA}^{i j}-\mathrm{CA}^{i j}\right)>\mathrm{x} \text {, then } \\
& \mathrm{ACA}^{i j}=\mathrm{ACA}^{i j}-\left(1-\gamma_{\mathrm{x}}\right),
\end{aligned}
$$

end if

$$
\text { If } \mathrm{ACA}^{i j}<\mathrm{Th} \text {, then }
$$

The flow is removed from the routing table. end if

Here $\gamma \in(0,1)$ represents a design parameter. $\mathrm{x}$ represents certain percentage.

\section{Simulation RESUlts}

\section{A. Simulation Model and Parameters}

We use NS2 [13] to simulate our proposed technique. In the simulation, the channel capacity of mobile hosts set to the same value: $11 \mathrm{Mbps}$. In the simulation, mobile nodes move in a 1000 meter $\mathrm{x} 1000$ meter region for 50 seconds simulation time. Initial locations and movements of the nodes are obtained using the random waypoint (RWP) model of NS2. It is assumed that each node moves independently with the same average speed. All nodes have the same transmission range of 250 meters. The node speed is $5 \mathrm{~m} / \mathrm{s}$. and pause time is 5 seconds.

TABLE 2. THE SIMULATION SETTINGS AND PARAMETERS

\begin{tabular}{|l|l|}
\hline No. of Nodes & 50 \\
\hline Area Size & 1000 X 1000 \\
\hline Mac & $802.11 \mathrm{e}$ \\
\hline Radio Range & $250 \mathrm{~m}$ \\
\hline Simulation Time & $50 \mathrm{sec}$ \\
\hline Routing Protocol & AODV \\
\hline Traffic Source & CBR and Video \\
\hline Video Trace & JurassikH263-256k \\
\hline Packet Size & 512 \\
\hline Mobility Model & Random Way Point \\
\hline Speed & $5 \mathrm{~m} / \mathrm{s}$ \\
\hline Pause time & $5 \mathrm{sec}$ \\
\hline MSDU & 2132 \\
\hline Rate & $50 \mathrm{~kb}, 100 \mathrm{~kb}, \ldots . .250 \mathrm{~Kb}$ \\
\hline No. of Flows & $4,5,6,7 \mathrm{and} 8$ \\
\hline Initial Energy & $5.1 \mathrm{~J}$ \\
\hline Transmit Power & $0.360 \mathrm{w}$ \\
\hline Receiving Power & $0.395 \mathrm{w}$ \\
\hline Idle Power & $0.335 \mathrm{w}$ \\
\hline
\end{tabular}

\section{B. Performance Metrics}

We compare the performance our Agent based Bandwidth Reservation (ABR) technique with the BRAWN [7] scheme. The performance is evaluated mainly, according to the following metrics.

- Aggregated Bandwidth: We measure the received bandwidth for class1 (VBR) and class2 (CBR) traffic of all flows
- Fairness Index: For each flow, we measure the fairness index as the ratio of throughput of each flow and total no. of flows

- Total Bandwidth: It is the sum of received bandwidth of class 1 and class 2 .

\section{RESULTS}

\section{A. Effect of Varying Rates}

In the initial experiment, we measure the performance of the proposed technique by varying the rate as 50,100, 150, 200and $250 \mathrm{~Kb}$.

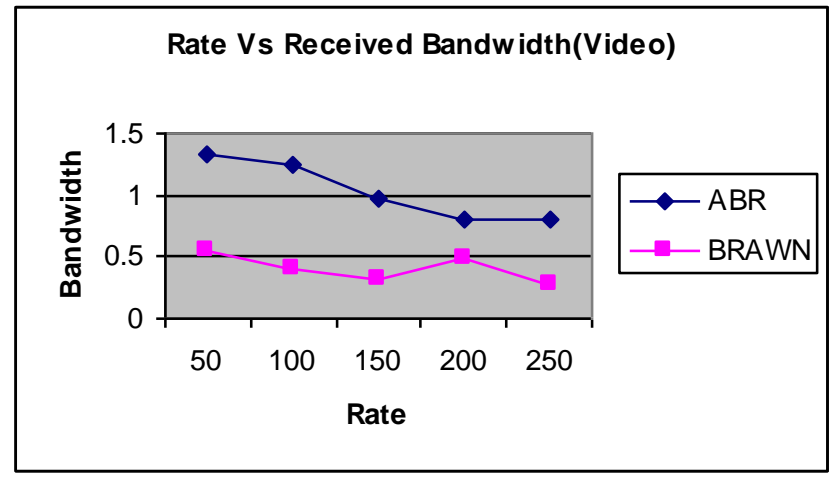

Fig 1: Rate Vs Received Bandwidth

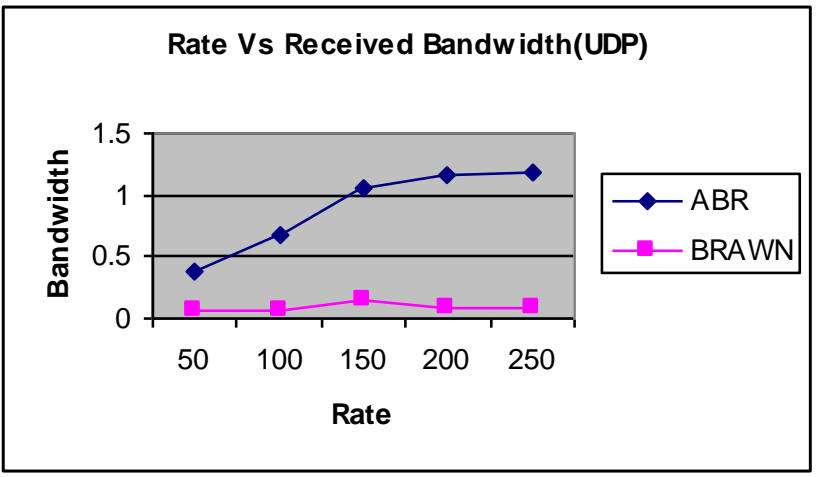

Fig 2: Rate Vs Received Bandwidth

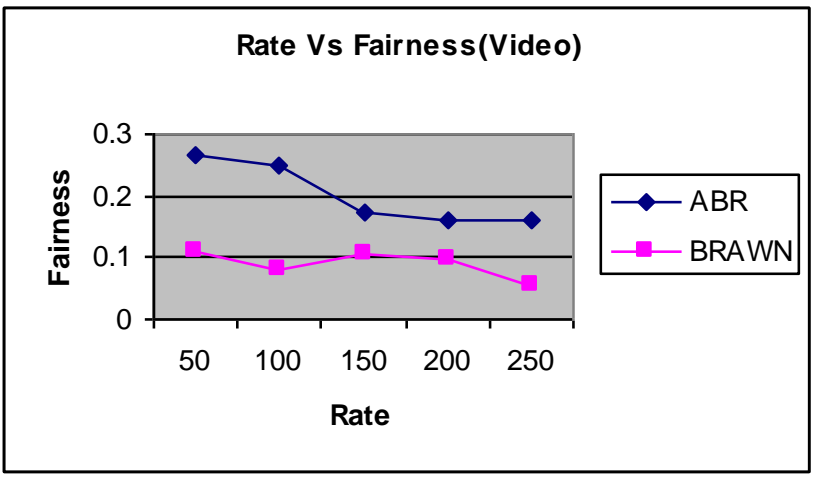

Fig 3: Rate Vs Fairness

Fig: 1 and Fig: 2 give the aggregated bandwidth for Video and UDP traffic. From the figures, it can be seen that ABR has received more bandwidth when compared with BRAWN. 
Fig: 3 and Fig: 4 give the fairness index for Video and UDP traffic. From the figures, it can be seen that ABR achieves more fairness when compared with BRAWN.

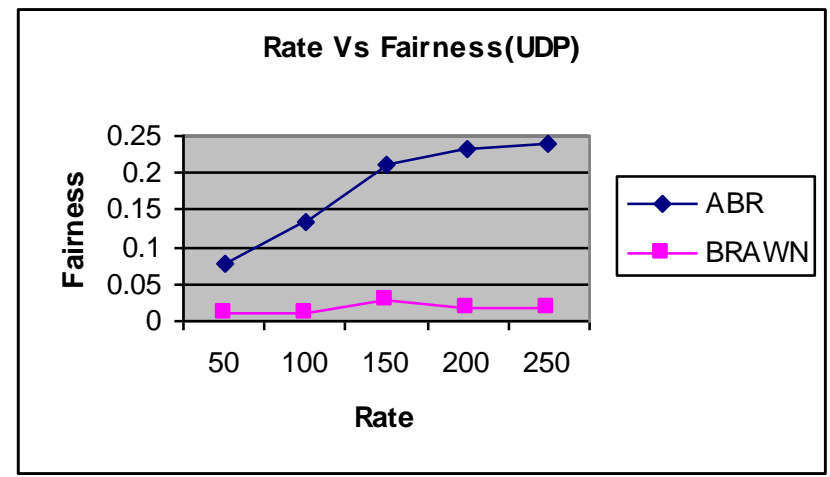

Fig 4: Rate Vs Fairness

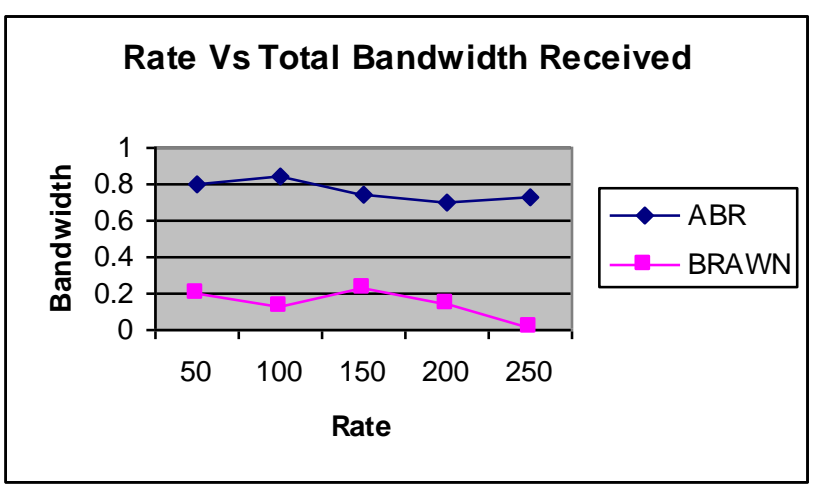

Fig 5: Rate Vs Total Bandwidth

Fig: 5 give the Total Bandwidth ratio. From figure, we can see that the proposed ABR has high total Bandwidth ratio than the BRAWN

\section{B. Effect of Varying Flows}

In the next experiment, we compare our proposed technique by varying the number of flows as 4,5,6,7 and 8 .

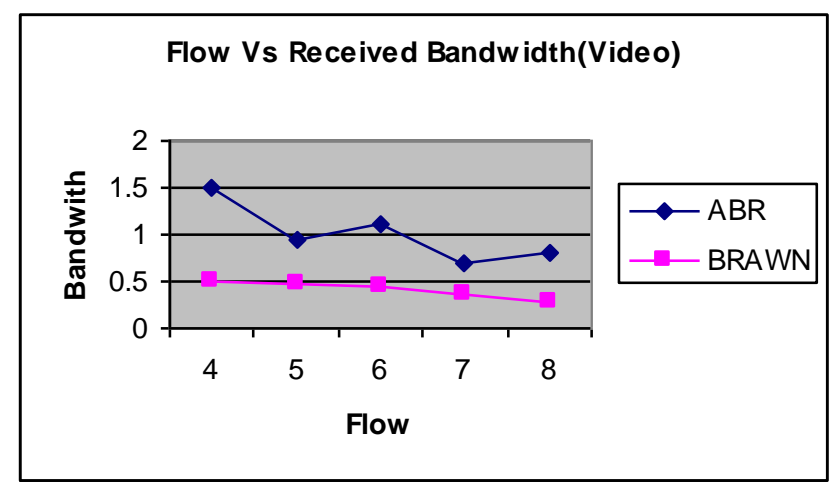

Fig 6: Flow Vs Received Bandwidth for VBR flows

Fig: 6 and Fig: 7 give the aggregated bandwidth for Video and UDP traffic. From the figures, it can be seen that ABR has received more bandwidth when compared with BRAWN.

Fig: 8 and Fig: 9 give the fairness index for Video and UDP traffic. From the figures, it can be seen that ABR achieves more fairness when compared with BRAWN.

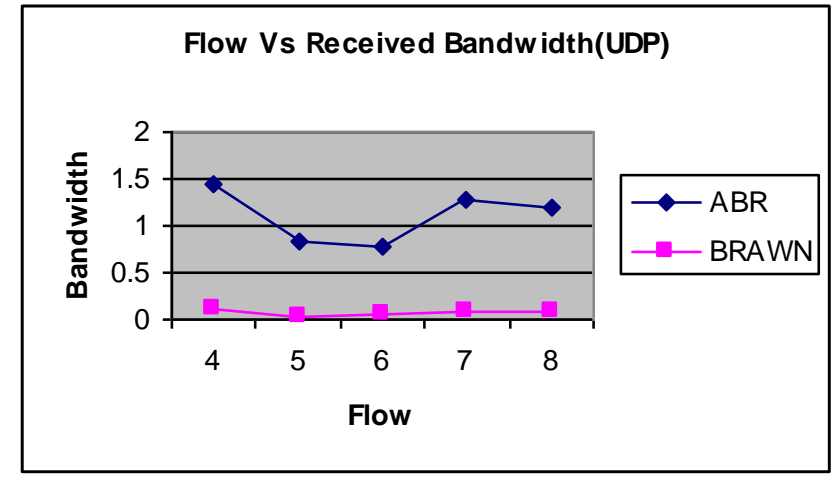

Fig 7: Flow Vs Received Bandwidth for CBR flows

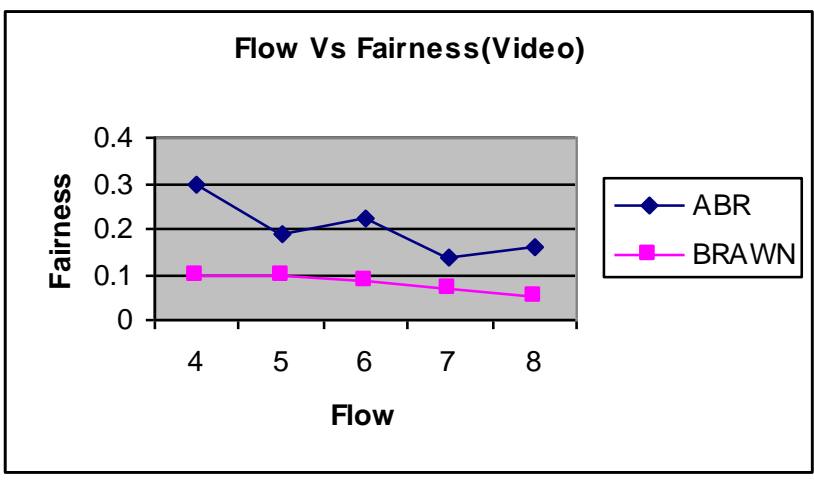

Fig 8: Flow Vs Fairness for VBR flows

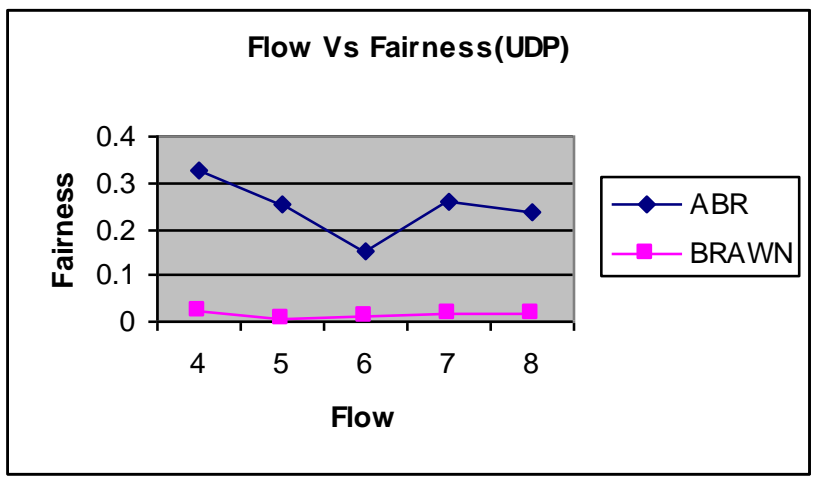

Fig 9: Flow Vs Fairness

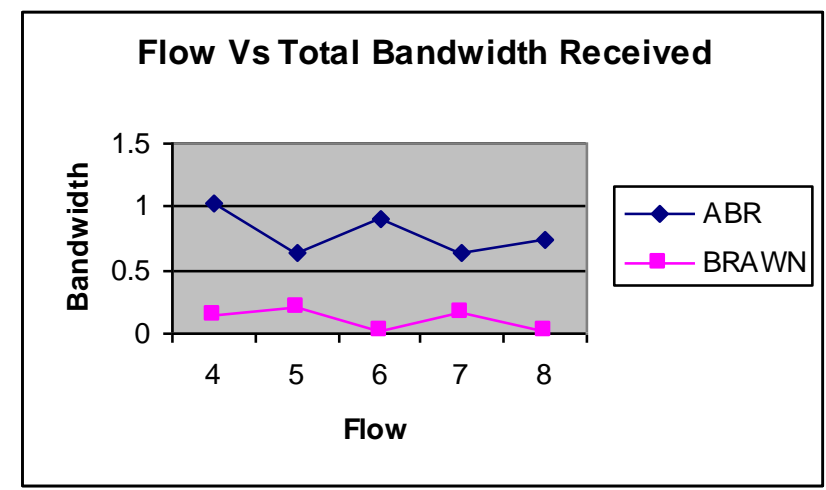

Fig 10: Flow Vs Total Bandwidth

Fig: 10 give the Total Bandwidth ratio. From figure, we can see that the proposed $\mathrm{ABR}$ has high total Bandwidth ratio than the BRAWN. 


\section{CONCLUSION}

In this paper, we have proposed an agent based bandwidth reservation technique for MANETs. The mobile agent from the source starts forwarding the data packets through the path which has minimum cost, congestion and bandwidth. The status of every node is collected which includes bottleneck bandwidth $\left(\mathrm{BW}_{\mathrm{BN}}\right)$ field and the intermediate node computes the available bandwidth $\left(\mathrm{B}_{\mathrm{av}}\right)$ on the link and finally the packet is intended to destination. After updating the new $\mathrm{BW}_{\mathrm{BN}}$ field, the data packet is feedback to the source. In resource reservation technique, if the $\mathrm{B}_{\mathrm{av}}$ is greater than $\mathrm{BW}_{\mathrm{BN}}$, then bandwidth reservation for the flow proceeds. Otherwise the $\mathrm{BW}_{\mathrm{BN}}$ field is overwritten with $\mathrm{B}_{\mathrm{av}}$ value. The rate control technique is added that contains traffic policing and rate monitoring and adjustment. The cumulative assigned rate of the incoming and outgoing flows helps in rate adjustments. By simulation results, we have shown that the resource allocation technique reduces the losses and improves the network performance.

\section{REFERENCES}

[1] S.Santhosh baboo and B.Narasimhan, "A Hop-by-Hop CongestionAware Routing Protocol for Heterogeneous Mobile Ad-hoc Networks", International Journal of Computer Science and Information Security, 2009

[2] A. Capone, J. Elias, F. Martignon, G. Pujolle, "Dynamic resource allocation in communication networks", in: Proceedings of Networking 2006, Coimbra, Portugal, 15-19 May 2006, also published in Springer LNCS, vol. 3976, 2006, pp. 892-903.

[3] Babbar, R., Fapojuwo, A., Far, B., "Agent-Based Resource Management in Hybrid Wireless Networks", IEEE Canadian Conference on Electrical and Computer Engineering, Vol. 3 (2004), pp. 1297-1300.

[4] C. Chaudet, I. Guérin Lassous and J. Zerovnik,"A distributed algorithms for bandwidth allocation in stable ad hoc networks", IFIP International Conference on Wireless On-Demand Network Systems (WONS), pp. 101-115, January 2004.

[5] R. Gunasekaran and V. Rhymend Uthariaraj, "Differentiated Bandwidth Allocation in Mobile Ad Hoc Networks (MANET) - A Profile Based Approach", Asian Journal of Information Technology, 2007.

[6] Kumar Manoj Member, IAENG, S. C. Sharma \& S.P. Singh," Dynamic Behavior of Bandwidth Control Management in Mobile Ad-Hoc Network ", Proceedings of the World Congress on Engineering and Computer Science, Vol I, 2009.

[7] Rafael Paoliello-Guimarães, Llorenç Cerdà, José M. Barceló, Jorge García-Vidal, Michael Voorhaen, Chris Blondia." Quality of service through bandwidth reservation on multirate ad hoc wireless networks Ad Hoc Networks", 388 400, 2009.
[8] Maria Canales , Jose Ramon, Gallego, Angela Hernandez Solana, Antonio Valdovinos, "QoS provision in mobile ad hoc networks with an adaptive cross-layer architecture", Journal Wireless Networks, Volume 15 Issue 8, November 2009.

[9] Wang Xiangli, Li Layuan, Gong Berican, Wang Wenbo, “ A Distributed Bandwidth Reservation Protocol for QoS Routing in Mobile Ad Hoc Networks", international conference on wireless communications,

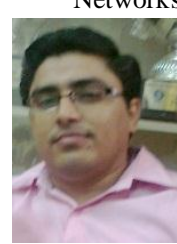
networking and mobile computing, vol 1-15, 2007.

[10] Binod Kumar Pattanayak, Manoj Kumar Mishra, Alok Kumar Jagadev, and Manoj Ranjan Nayak,” A Cluster-based QoS Support To Bandwidth Preservation With Concept Of Survivability In Multi-hop Mobile Ad Hoc Networks", Proceedings of The World Congress on Engineering and Computer Science , pp247-254, 2009.

[11] Bhadauria SS,Sharma V.,Framework and Implimentation of an Agent Based Congestion Control Technique for Mobile Ad-hoc Network ,ICAC3 2011, CCIS, Volume 125, Springer,pp. 318-327, 2011.

[12] Sharma V, Bhadauria SS," Agent Based Congestion Control Routing for Mobile Ad-hoc Network”Wimon-2011,CCIS,Springer,Volume 197, pp.324-333,2011.

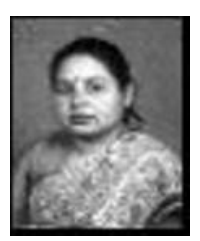

[13] Network Simulator, http://www.isi.edu/nsnam/ns

[14] Vishnu Kumar Sharma1 and Dr. Sarita Singh Bhadauria. Congestion and Power Control Technique Based on Mobile Agent and Effect of Varying Rates in MANET. IJACSIT Vol 1 No 1.

Vishnu Sharma, working as a faculty in CSE Dept. Of Jaypee University of Engg and Technology and presently, he is a Ph.D Candidate at M.P.Technical University, He has about 13 years of teaching experience. He has published more than fifteen papers in the area of Mobile Ad-hoc Networks \& Mobile Computing at National/International Level. He has published the books on Mobile Computing and Advanced Mobile Computing in UPTU, Lucknow (UP) and IP University, New Delhi. He is a life time member of International Association of Computer Science and Information Technology (IACSIT), Singapore and member of CSI. His area of interest includes Mobile Computing, Computer Networks and Communication Technologies, Cryptography and Network Security.

Dr.Sarita Singh Bhadauria She is a Professor in Department of Electronics MITS, Gwalior (M.P.) India; she has about 25 years of teaching and research experience. She has published more than fifty papers in the area of Digital Image Processing, Computer Networks, Mobile Ad-hoc Networks, Mobile Communication and Cryptography and Network Security and Digital Communication at National/International Level. She is a life time member of ISTE, IETE, IEEE, CSI and HAM RADIO. Her areas of interest include Wireless Communication and Digital Image Processing, Computer Networks, and Mobile Ad-hoc Networks, Mobile Communication and Cryptography and Network Security and Digital Communication and Communication Technologies. 